\title{
Caracterização do Nível Tecnológico dos Apicultores do Estado do Rio de Janeiro
}

\author{
Niraldo José Ponciano ${ }^{1}$, Adelmo Golynski², \\ Paulo Marcelo de Souza ${ }^{3}$, Marlon Gomes $\mathrm{Ney}^{4}$ e \\ Vanuza da Silva Pereira Ney ${ }^{5}$
}

Resumo: $\mathrm{O}$ estudo analisa e busca subsídios para gerar e transferir tecnologias compatíveis com o nível socioeconômico dos apicultores. Os dados foram extraídos a partir do Censo Apícola, englobando 75 municípios com atividade apícola representativa. Foi utilizada a Análise Fatorial para caracterizar o nível tecnológico dos apicultores. Os resultados evidenciaram baixo nível tecnológico. Por meio dos escores fatoriais calculou-se o índice bruto de desenvolvimento. Com valores mais altos desse índice, destacaram-se os municípios de Conceição de Macabu, Areal, Duas Barras, Rio Claro, Cardoso Moreira, Cachoeiras de Macacu, Campos dos Goytacazes, Natividade e Nova Iguaçu. Constatou-se que o grau de escolaridade influenciou positivamente o desenvolvimento dos apicultores. Esse desenvolvimento também foi afetado pela assistência técnica, pelo manejo da troca de rainha e pela prática da apicultura migratória. Dessa forma, a modernização dos apicultores no sentido de melhorar o nível tecnológico, expandir a produtividade e diversificar a produção passa necessariamente pelo nível de conhecimento do apicultor e pela situação socioeconômica.

Palavras-chaves: Apicultura, análise fatorial, nível tecnológico.

Abstract: The study examines and seeks grants to generate and transfer compatible technologies with the socioeconomic status of beekeepers. Data were extracted from the Census Beekeeping, involving 75 municipalities with significant beekeeping activity. Factor analysis was used to characterize the technological level of beekeepers. The results showed low technological level. Using the factor scores the gross rate of development was calculated. With higher values of this index, Conceição de Macabu, Areal, Duas Barras, Rio Claro, Cardoso Moreira, Cachoeiras de Macacu, Campos dos Goytacazes, Natividade e Nova Iguaçu cities were highlighted. It was found that the level of education has affected positively the development of beekeepers. This development was also affected

\footnotetext{
1 Professor da Universidade Estadual do Norte Fluminense. Campos (RJ). E-mail: ponciano@uenf.br

2 Professor do Instituto Federal de Morrinhos (GO). E-mail: agolynski@yahoo.com.br

3 Professor da Universidade Estadual do Norte Fluminense. Campos (RJ). E-mail: pmsouza@uenf.br

4 Professor da Universidade Estadual do Norte Fluminense. Campos (RJ). E-mail: marlonney@uenf.br

5 Professora da Universidade Federal Fluminense. Campos (RJ). E-mail: vanuzap@hotmail.com
} 
by the technical assistance, by the management and exchange of queen practice and by the migratory beekeeping. Thus, the modernization of beekeepers to improve the technological level, expand productivity and diversify their production necessarily involves knowledge level of the beekeeper and the socioeconomic situation.

Key-words: Beekeeping, factor analysis, technological level.

Classificação JEL: C38, Q16, R11.

\section{Introdução}

A atividade apícola teve início no Brasil com a introdução de abelhas da espécie Apis mellifera no estado do Rio de Janeiro, em 1839, pelo padre Antônio Carneiro, trazidas da região do Porto, em Portugal (SOUZA, 2011). Segundo o mesmo autor, em 1956, alguns enxames de abelha africana (Apis Mellifera Scutellata) escaparam do apiário experimental e cruzaram com as de raças europeias, formando um híbrido natural produtivo.

Os principais produtos obtidos e comercializados da atividade apícola são: mel, cera, própolis, geleia real e apitoxina. $\mathrm{O}$ mel pode ser considerado o produto apícola mais fácil de ser explorado, mais conhecido, e com maiores possibilidades de comercialização, seja como fonte alimentar ou insumo de indústrias farmacêuticas e cosméticas.

Os programas de incentivo à produção e capacitação de apicultores têm contribuído para o crescimento do setor. Enquanto no Brasil a produtividade média de uma colmeia é de cerca de 15 quilos de mel por ano, na Argentina, alcança 35 quilos e, na China, uma colmeia chega a produzir 100 quilos de mel por ano. Muito embora a produtividade brasileira ainda seja considerada pequena, princi- palmente devido ao baixo nível tecnológico utilizado nos apiários, percebe-se que existe potencial elevado.

Segundo dados do IBGE (Instituto Brasileiro de Geografia e Estatística), o Brasil produziu, de 2008 a 2010, uma média de 38,26 mil toneladas de mel, ocupando a $11^{\text {a }}$ posição no ranking dos produtores mundiais, tornando-se o quinto maior exportador. A cadeia produtiva da apicultura envolve cerca de 350 mil pessoas no País, sendo a maioria agricultores familiares.

Baseado na mesma fonte e considerando a produção média do período de 2001 a 2010, o Brasil produziu 32,80 mil toneladas de mel. Os cinco maiores estados produtores foram responsáveis por $64 \%$ e os dez maiores, por $90 \%$. O maior produtor de mel nacional é o Rio Grande do Sul, com mais de 7 mil toneladas por ano, em média, no período de 2001 a 2010. Em segundo lugar, está o Paraná, com média de mais de 4 mil toneladas. Para completar os dez maiores estados produtores, destacam-se: Santa Catarina, Piauí, Minas Gerais, Ceará, São Paulo, Bahia, Pernambuco e Rio Grande do Norte.

Os dados do IBGE apontam que o estado do Rio de Janeiro produziu, em média, 350 toneladas de mel por ano no período de 2001 a 
2010, ocupando, assim, a 13ª colocação, atrás de Maranhão e Mato Grosso do Sul. Analisando-se historicamente a produção do estado, percebe-se grande evolução na década de 1980 (Figura 1). A produção do estado, que no início da referida década era de cerca de 45 toneladas, encerrou essa década com 368 toneladas de mel por ano. Tal expansão pode ser atribuída à difusão de tecnologia pelos órgãos de extensão rural, que permitiu a utilização adequada do manejo de grande parte dos apicultores. $\mathrm{O}$ ápice de produção ocorreu em 1995, com mais de 500 toneladas, ocorrendo, a partir de então, decréscimo constante da produção de mel.

A apicultura pode ser considerada alternativa importante de geração de renda para agricultores familiares. É uma atividade de fácil manutenção, baixo custo inicial e sustentável do ponto de vista social, econômico e ambiental. Possibilita a ocupação no campo, além da obtenção de renda extra a baixo custo. As abelhas atuam como polinizadoras naturais, preservando espécies e contribuindo para o equilíbrio do ecossistema e manutenção da biodiversidade.

A atividade rural do Rio de Janeiro encontra-se baseada em um número limitado de produtos, sendo esta uma característica bastante indesejável sob o aspecto da sustentabilidade do desenvolvimento econômico, devido à pequena diversificação das atividades rurais. A apicultura apresenta-se como uma alternativa para aumentar a diversificação da agropecuária.

Nesse contexto, tal potencial pode ser alcançado com geração e adoção de tecnologia pelos apicultores. Mesmo que a adoção de inovações tecnológicas promova o crescimento dos níveis de produtividade, parte dos produtores não o faz por fatores socioeconômicos relacionados (FREITAS, KHAN e SILVA, 2004). A pesquisa sobre análise de mudança tecnológica e socioeconômica tem se constituído em um processo que envolve inovação, difusão tecnológica e condições sociais dos adotantes, modificando a concepção da relação existente entre a inovação e a difusão de novos processos (ROGERS, 2003). Na visão moderna de mudança tecnológica, não se separam inovação, difusão e condições socioeconômicas como temas distintos, pois eles são considerados como inter-relacionados.

Acredita-se que a modernização da apicultura no sentido de melhorar o nível tecnológico e expandir a produtividade passa, necessariamente, pelo nível de conhecimento do agricultor e sua situação socioeconômica. Enfatiza-se que o

Figura 1. Comportamento da produção de mel no estado do Rio de Janeiro no período de 1974 a 2010, em toneladas

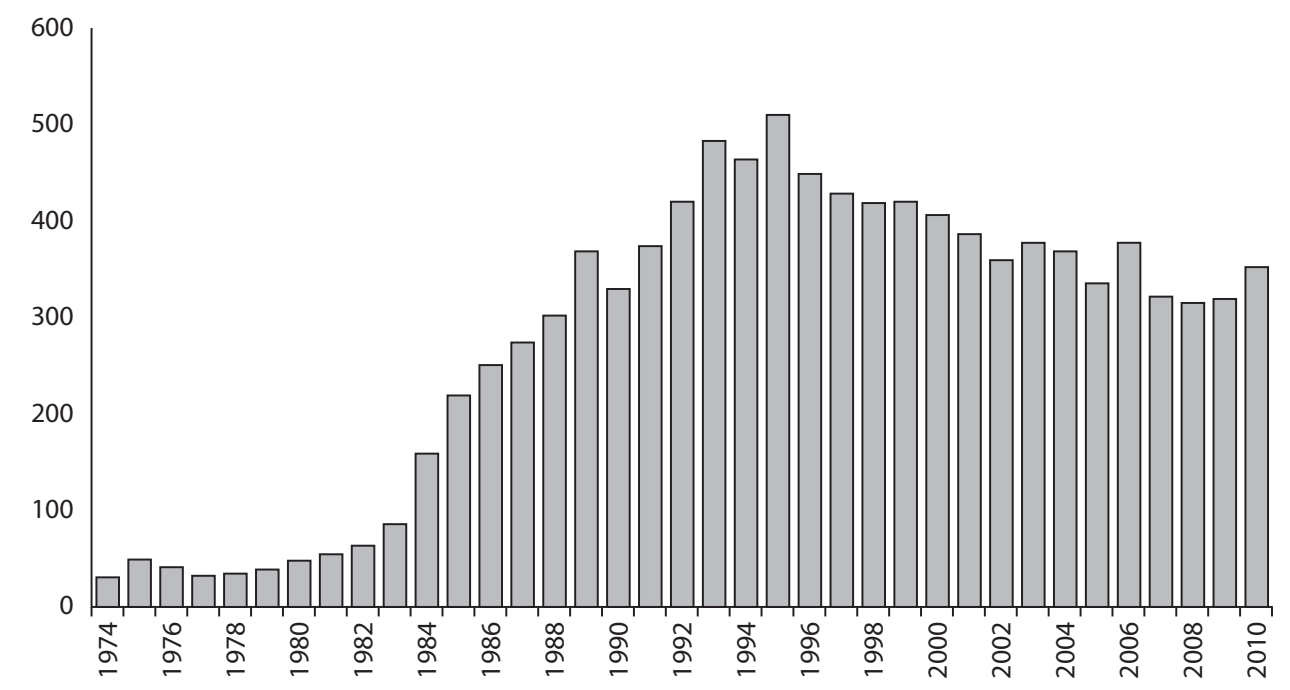

Fonte: IBGE. 
baixo nível tecnológico do apicultor fluminense contrasta com o nível de conhecimentos produzidos pelos órgãos de pesquisa. Para conhecer a realidade e encontrar subsídios para gerar e transferir tecnologias compatíveis com essa realidade é importante ter conhecimento do perfil desses apicultores.

O objetivo do presente estudo foi caracterizar o nível tecnológico dos produtores de mel do estado do Rio de Janeiro. Especificamente, buscou-se classificar os municípios segundo o grau de utilização de tecnologia de sua apicultura, bem como analisar a influência do nível educacional do apicultor no nível tecnológico empregado.

\section{Fundamentação teórica}

A interação social e a troca de experiências são fundamentais no processo de transferência de tecnologia e de inovação tecnológica. Segundo Tornatzy (1990), a inovação tecnológica envolve tanto situações de novos desenvolvimentos como introdução de novos conhecimentos. A competitividade de um produtor relaciona-se tanto com sua capacidade de adaptabilidade e flexibilidade de produção quanto com a competitividade sistêmica dos demais integrantes da cadeia produtiva. Para Blake e Saleh (1992), inovação é a estratégia de explorar oportunidades de mercado, introduzindo ou adaptando novos produtos. Assim, a adaptabilidade à dinâmica do mercado passa necessariamente pela inovação tecnológica.

Analisando competitividade e nível tecnológico da apicultura no estado do Ceará, Khan et al. (2009) constataram que o nível tecnológico foi determinante na competitividade dos apicultores, sendo que a maior contribuição foi da tecnologia de colheita e a menor, da tecnologia de gestão. Os autores concluíram que os principais condicionantes do nível tecnológico dos apicultores foram o grau de instrução, o objetivo com a atividade e o acesso à assistência técnica e ao crédito.

Com a finalidade de caracterizar sistemas de produção de leite em Minas Gerais, Chinelatto
Neto et al. (2005) utilizaram Análise Fatorial para descrever um conjunto de variáveis a partir de um número menor de fatores, e análise de cluster para classificação em três grupos de produtores homogêneos.

Carvalho et al. (2007) analisaram o desempenho competitivo das empresas da indústria de móveis de madeira do Pará por meio do modelo de Análise Fatorial. Os autores mostraram que a maioria das empresas da indústria de móveis apresentou desempenho competitivo intermediário, em função das limitações de fatores como a falta de mão de obra qualificada, baixo nível tecnológico, dificuldade de acesso ao crédito e baixo grau de integração da cadeia de suprimento.

Para caracterizar o grau de desenvolvimento tecnológico das regiões norte e noroeste do estado do Rio de Janeiro, Souza et al. (2009) empregaram a técnica da Análise Fatorial a um conjunto de variáveis relacionadas à utilização de tecnologias modernas. Os autores descreveram o processo de modernização nos municípios e, mediante a Análise de Cluster, os agruparam em classes distintas quanto à intensidade da modernização atingida.

O método da Análise Fatorial refere-se a um conjunto de técnicas estatísticas cujo objetivo comum é representar um grupo de variáveis em termos de um menor número de variáveis hipotéticas (KIM e MUELLER, 1978). De acordo com Schilderinck (1970), essa técnica consiste na tentativa de se determinar as relações quantitativas entre as variáveis, aferindo seus padrões de movimento, de modo a associar, àquelas com padrão semelhante, o efeito de um fator causal subjacente e específico a estas variáveis. Segundo Johnson e Wichern (1992), essa técnica se baseia na suposição da existência de um número de fatores causais gerais, cuja presença dá origem às relações entre as variáveis observadas, de forma que, no total, o número de fatores é consideravelmente inferior ao número de variáveis. Isso decorre do fato de que muitas relações entre as variáveis são, em grande parte, devidas ao mesmo fator causal geral. 


\section{Metodologia}

\subsection{Emprego da Análise Fatorial}

A técnica da Análise Fatorial foi utilizada com a finalidade de descrever o processo de adoção de tecnologia na apicultura, sintetizando, em um número reduzido de fatores, as diversas tecnologias empregadas. Para melhor interpretação, procedeu-se a uma rotação ortogonal dos fatores, com o método Método Varimax, sugerido por Kim e Mueller (1978). Com este procedimento, a contribuição de cada fator para a variância total foi alterada sem modificar a contribuição conjunta deles. Como vantagem, os fatores obtidos após a rotação se encontraram mais estreitamente relacionados a determinados grupos de variáveis, possibilitando melhor interpretação destes.

As variáveis foram normalizadas para possibilitar melhor comparação entre elas. A normalização consistiu em expressar, em desvios padrão, os desvios das observações originais em relação à média. Cada variável normalizada $z_{i}(i=1,2$, $\ldots, n)$ foi relacionada separadamente aos fatores $f_{j}$ $(j=1,2, \ldots, m),(m<n, N)$. As relações são lineares e assumem, no modelo básico de Análise Fatorial, a seguinte expressão analítica, conforme Harman (1967):

$z_{i}=a_{i 1} f_{1}+a_{i 2} f_{2}+\ldots+a_{i m} f_{m}+d_{i} u_{i} \quad(i=1,2, \ldots, n)$

em que cada uma das n variáveis é descrita, em termos lineares, como função dos $m$ fatores comuns $f_{j}(j=1,2, \ldots, m)$, aos quais se relacionam por meio das cargas fatoriais ou coeficientes de conexão $a_{i j}$, que indicam em que medida e direção as variáveis $z_{i}$ estão relacionadas com o fator $f_{j}$; e de um fator único $u_{i}$, que responde pela variância remanescente.

Para saber se os fatores gerais causaram alguma relação entre as variâncias de $z_{i}$, foi necessário que sua variância total $\left(\sigma_{i}^{2}\right)$ fosse dividida em três componentes:

1. A variância comum ou comunalidade, $h_{i}^{2}$, que indica a proporção da variância total de $z_{i}$ está associada com a variância das outras variáveis.
2. A variância específica ou especificidade, $S_{i}{ }^{2}$, isto é, a proporção da variância total que não mostra qualquer associação com a variância das outras variáveis; e

3. O erro ou disturbância, $e_{i}^{2}$, que é a proporção da variância devida aos erros nas observações, ou a variáveis relevantes ao estudo.

Os fatores únicos que não são correlacionados com os fatores comuns com variância total de $z_{i}, \sigma_{i}^{2}$ podem ser expressos por:

$$
\sigma_{i}^{2}=a_{i 1}^{2}+a_{i 2}^{2}+\ldots+a_{i m}^{2}+d_{i}^{2}
$$

em que os componentes $a_{i j}{ }^{2}$ são as percentagens de conexão e correspondem à proporção da variância total da variável normalizada $z_{i}$ que é explicada pelos respectivos fatores. Assim, na expressão (2), o termo:

$$
h_{i}^{2}=a_{i 1}^{2}+a_{i 2}^{2}+\ldots+a_{i m}^{2}
$$

equivale à comunalidade da variável $z_{i}$, ao passo que o termo $d_{i}^{2}$ corresponde à unicidade e indica a extensão em que os fatores comuns falham na explicação da variância total da variável.

A unicidade foi decomposta em duas partes: uma referente à seleção das variáveis, denominada especificidade $\left(s_{i}^{2}\right)$, e outra atribuída à não confiabilidade das medidas, denominada erro $\left(e_{i}^{2}\right)$ :

$$
d_{i}^{2}=s_{i}^{2}+e_{i}^{2}
$$

com essa decomposição, o modelo linear (4) passa a ter a seguinte forma:

$z_{i}=a_{i 1} f_{1}+a_{i 2} f_{2}+\ldots+a_{i m} f_{m}+b_{i} s_{i}+c_{i} e_{i} \quad(i=1,2, \ldots, n)$

em que $s_{i}$ e $e_{i}$ são os fatores específico e erro, respectivamente, e $b_{i}$ e $c_{i}$ são seus coeficientes.

\section{2. Índice bruto de desenvolvimento}

Uma vez obtidos os fatores e correspondentes escores fatoriais, a classificação dos municípios segundo o grau de desenvolvimento tecnológico foi realizada mediante o emprego do Índice Bruto de Desenvolvimento (IB). Esse índice, uti- 
lizado por Melo e Parré (2007), é calculado com base na média ponderada dos escores fatoriais obtidos. Para um conjunto de $n$ fatores e, consequentemente, $n$ escores fatoriais, o índice bruto de modernização é obtido por:

$$
I B=\frac{\sum_{i=1}^{n} w i F i}{\sum_{i=1}^{n} w i}
$$

em que wi é a proporção da variância explicada por fator e Fi o valor dos escores fatoriais.

O procedimento seguinte consistiu na obtenção dos escores fatoriais, utilizados para classificar os municípios conforme o grau de adoção de tecnologia na apicultura. Tal classificação foi realizada com o uso do índice bruto de desenvolvimento, obtido a partir dos escores fatoriais, conforme metodologia proposta por Melo e Parré (2007).

\subsection{Variáveis e fonte dos dados}

Os fatores foram determinados por meio da seleção de 13 variáveis relativas ao uso de tecnologia na apicultura, conforme descrito a seguir, com a finalidade de caracterizar o grau de desenvolvimento tecnológico dos apicultores para os 75 municípios que apresentaram atividade apícola representativa no estado.

- $\mathrm{X} 1=\%$ de produção de cera por apicultor;

- $\mathrm{X} 2=\%$ de produção de mel por apicultor;

- $\mathrm{X} 3=\%$ de apicultores que realizam a troca de rainhas;

- X4 = \% de produção de própolis por apicultor;

- X5 $=\%$ de apicultores que praticam apicultura migratória;

- X6 = \% de produção de geleia real por apicultor;

- $\mathrm{X} 7=\%$ de apicultores que não apresentam uso de agrotóxico num raio de $3 \mathrm{~km}$;

- $\mathrm{X} 8=\%$ de apicultores que recebem assistência técnica;

- X9=\% de produção de pólen por apicultor;

- $\mathrm{X} 10=\%$ de apicultores que utilizam abelhas não africanizadas;
- $\mathrm{X} 11=\%$ de apicultores que praticam apicultura fixa;

- $\mathrm{X} 12=\%$ de apicultores que deram origem aos enxames por meio da multiplicação;

- $\mathrm{X} 13=\%$ de apicultores que não apresentaram perdas de colmeias;

A fonte dos dados utilizados foi o Censo Apícola $^{6}$ do estado do Rio de Janeiro, realizado pelo Serviço Nacional de Aprendizagem do Cooperativismo no Estado do Rio de Janeiro (2006), com o apoio de várias instituições. Os dados foram obtidos por meio de entrevistas e questionários aplicados aos apicultores e representantes de cooperativas, entrepostos e associações. Sua abrangência englobou 75 municípios que apresentaram atividade apícola representativa no estado do Rio de Janeiro.

\section{Resultados e discussão}

A partir das informações disponíveis, foram extraídos, pelo método dos componentes principais, quatro fatores com raízes características maiores que um (Tabela 1). Optou-se pela caracterização no uso de tecnologias para os apicultores considerados por meio dos quatro primeiros fatores, que explicam $75 \%$ da variância total das variáveis analisadas.

Para facilitar a interpretação dos fatores, estes foram submetidos à rotação ortogonal pelo Método Varimax, sugerido por Kim e Mueller (1978). Com esse procedimento, a contribuição de cada fator para a variância total é alterada sem modificar a contribuição conjunta deles. Como vantagem, os fatores obtidos após a rotação se encontram mais estreitamente relacionados a determinados grupos de variáveis, possibilitando melhor interpretação.

As cargas fatoriais, as comunalidades e o percentual da variância total dos indicadores expli-

6 Os autores agradecem à Prof. Maria Cristina Affonso Lorenzon (UFRRJ) e ao Serviço Nacional de Aprendizagem do Cooperativismo no estado do Rio de Janeiro pela disponibilização dos dados e informações do Censo Apícola do estado. 
Tabela 2. Fatores obtidos pelo método dos componentes principais

\begin{tabular}{cccc}
\hline Fator & $\begin{array}{c}\text { Raiz } \\
\text { Característica }\end{array}$ & $\begin{array}{c}\text { Variância Explicada } \\
\text { pelo Fator (\%) }\end{array}$ & $\begin{array}{c}\text { Variância } \\
\text { Acumulada (\%) }\end{array}$ \\
\hline 1 & 4,63 & 35,59 & 35,59 \\
2 & 2,06 & 15,87 & 51,45 \\
3 & 1,84 & 14,17 & 65,62 \\
4 & 1,16 & 8,95 & 74,57 \\
\hline
\end{tabular}

Fonte: Dados da pesquisa, baseado no Censo Apícola do estado do Rio de Janeiro (2006).

cados por fator, após a rotação, encontram-se na Tabela 2. Feita a rotação, a contribuição dos fatores F1, F2, F3 e F4 para explicação da variância total dos indicadores utilizados passa a ser $33,80 \%$, $16,19 \%, 14,59 \%$ e $9,98 \%$, respectivamente, com contribuição conjunta de $74,57 \%$.

As cargas fatoriais com valor superior a 0,600 (em negrito) evidenciam os indicadores mais fortemente associados a determinado fator. Pode-se constatar que o fator 1 está mais estreitamente relacionado às variáveis $\mathrm{X} 3, \mathrm{X} 4, \mathrm{X} 8, \mathrm{X} 9$ e $\mathrm{X} 11$. O segundo fator encontra-se mais fortemente correlacionado com a variável $\mathrm{X} 13$. O terceiro fator está correlacionado com as variáveis $\mathrm{X} 1, \mathrm{X} 2$ e X5. $\mathrm{O}$ quarto fator está mais fortemente correlacionado à variável $\mathrm{X} 6$.

Obtidos os fatores de modernização, a etapa seguinte consistiu em obter os escores fatoriais, segundo Monteiro e Pinheiro (2004), cujos valores encontram-se na Tabela 3.

Tabela 3. Cargas fatoriais após a rotação ortogonal e comunalidades obtidas na Análise Fatorial dos indicadores de modernização da apicultura do Rio de Janeiro

\begin{tabular}{|c|c|c|c|c|c|}
\hline \multirow{2}{*}{ Variável } & \multicolumn{4}{|c|}{ Fatores } & \multirow{2}{*}{ Comunalidades } \\
\hline & F1 & F2 & F3 & F4 & \\
\hline $\mathrm{X} 1$ & $-0,117$ & $-0,145$ & 0,883 & 0,063 & 0,538 \\
\hline $\mathrm{X} 2$ & $-0,161$ & $-0,155$ & 0,682 & 0,315 & 0,600 \\
\hline $\mathrm{X} 3$ & 0,929 & 0,110 & $-0,029$ & $-0,189$ & 0,916 \\
\hline$X 4$ & 0,967 & 0,069 & $-0,018$ & $-0,078$ & 0,922 \\
\hline X5 & 0,029 & 0,171 & 0,755 & $-0,153$ & 0,949 \\
\hline X6 & $-0,090$ & 0,294 & 0,015 & 0,882 & 0,790 \\
\hline $\mathrm{X} 7$ & 0,409 & $-0,170$ & $-0,196$ & 0,096 & 0,760 \\
\hline $\mathrm{X} 8$ & 0,743 & $-0,122$ & $-0,101$ & $-0,087$ & 0,857 \\
\hline X9 & 0,953 & 0,128 & $-0,030$ & 0,152 & 0,945 \\
\hline $\mathrm{X} 10$ & 0,035 & 0,522 & $-0,152$ & $-0,513$ & 0,830 \\
\hline $\mathrm{X} 11$ & 0,877 & 0,156 & $-0,039$ & $-0,120$ & 0,951 \\
\hline $\mathrm{X} 12$ & 0,171 & $-0,897$ & $-0,041$ & $-0,124$ & 0,954 \\
\hline $\mathrm{X} 13$ & 0,349 & 0,876 & $-0,075$ & 0,138 & 0,906 \\
\hline \% Variância & $33,80 \%$ & $16,19 \%$ & $14,59 \%$ & $9,98 \%$ & \\
\hline
\end{tabular}

Fonte: Dados da pesquisa, baseado no Censo Apícola do estado do Rio de Janeiro (2006). 
Tabela 4. Escores fatoriais obtidos por municípios que apresentaram atividade apícola

\begin{tabular}{|c|c|c|c|c|}
\hline \multirow{2}{*}{ Municípios } & \multicolumn{4}{|c|}{ Escores } \\
\hline & $F 1$ & $F 2$ & F3 & $F 4$ \\
\hline Casimiro de Abreu & 0,14634 & 0,25099 & $-0,11833$ & 1,19397 \\
\hline Rio das Ostras & 0,97612 & 0,09256 & 0,64764 & $-0,90925$ \\
\hline Silva Jardim & $-0,19208$ & $-0,10208$ & $-0,95564$ & $-0,3684$ \\
\hline Araruama & $-0,36033$ & $-0,11935$ & $-0,79065$ & $-0,36989$ \\
\hline Cabo Frio & 0,72883 & 0,85974 & $-0,35484$ & $-0,98861$ \\
\hline Iguaba Grande & $-0,24708$ & $-0,96519$ & $-1,09372$ & $-0,41461$ \\
\hline São Pedro da Aldeia & $-0,17173$ & $-0,83418$ & $-0,99837$ & $-0,22785$ \\
\hline Saquarema & $-0,47835$ & 1,85588 & $-0,50118$ & 0,57366 \\
\hline Carmo & 0,53534 & 0,43372 & $-0,1086$ & 0,68728 \\
\hline Cordeiro & $-0,83509$ & $-1,42224$ & 0,15877 & 0,09131 \\
\hline Macuco & $-0,91717$ & $-1,33426$ & $-0,75145$ & $-0,60158$ \\
\hline Bom Jardim & $-1,0747$ & $-1,3314$ & $-0,70829$ & $-0,17432$ \\
\hline Duas Barras & 0,29145 & $-0,28063$ & 6,4854 & $-0,17928$ \\
\hline Nova Friburgo & $-0,99177$ & $-0,32289$ & $-0,32353$ & $-0,17759$ \\
\hline Sumidouro & $-1,03134$ & $-0,63457$ & $-0,79897$ & $-0,59459$ \\
\hline Santa Maria Madalena & $-0,59474$ & $-0,85726$ & $-0,62939$ & $-0,24517$ \\
\hline Trajano de Morais & $-0,21362$ & $-0,53391$ & 0,01617 & $-0,45316$ \\
\hline Areal & 3,86225 & 0,90205 & $-0,48084$ & $-1,9159$ \\
\hline Comendador Levy Gasparian & 0,0527 & $-0,17136$ & 1,13859 & 0,52358 \\
\hline Paraíba do Sul & $-0,17603$ & 0,90492 & 1,28875 & $-0,59185$ \\
\hline Sapucaia & $-0,17354$ & $-0,45607$ & $-0,0394$ & 0,75029 \\
\hline Três Rios & $-0,11470$ & $-0,80228$ & 0,22229 & 0,56495 \\
\hline Itaguaí & 0,02846 & 0,16477 & $-0,52487$ & $-0,26906$ \\
\hline Mangaratiba & 1,08605 & $-0,42262$ & $-0,95925$ & $-1,46163$ \\
\hline Seropédica & 0,61909 & 0,09991 & 0,69326 & $-0,04324$ \\
\hline Cachoeiras de Macacu & 2,78488 & $-1,88902$ & 0,37120 & 0,18992 \\
\hline Rio Bonito & 0,10951 & $-1,05177$ & 0,70651 & $-0,20262$ \\
\hline Duque de Caxias & $-1,89265$ & 3,84505 & $-0,82539$ & $-2,86782$ \\
\hline Itaboraí & $-0,72296$ & $-0,38593$ & 0,99575 & 0,34610 \\
\hline Japeri & $-0,63538$ & 1,77591 & 1,83854 & $-1,14877$ \\
\hline Magé & $-1,20849$ & $-0,60154$ & 0,10712 & $-0,77114$ \\
\hline Maricá & $-0,31672$ & $-0,23309$ & 0,40972 & $-0,0466$ \\
\hline Niterói & $-0,03299$ & 0,96867 & 0,32021 & 0,64256 \\
\hline Nova Iguaçu & $-0,76777$ & 2,67663 & $-1,24635$ & 4,12996 \\
\hline Rio de Janeiro & $-0,09034$ & 0,53044 & 0,27695 & 0,45795 \\
\hline São Gonçalo & $-0,88285$ & $-0,74992$ & $-0,56065$ & $-0,05518$ \\
\hline Tinguá & $-0,55682$ & $-0,09829$ & 0,9636 & 0,07725 \\
\hline Petrópolis & $-0,3734$ & $-0,07253$ & 1,23017 & 0,61071 \\
\hline Teresópolis & $-1,11084$ & $-0,6566$ & $-0,88277$ & $-0,41269$ \\
\hline Engo Paulo de Frontin & $-0,11577$ & $-0,53469$ & $-0,13249$ & 0,14251 \\
\hline Mendes & $-0,71841$ & 0,66781 & 0,12045 & $-0,43114$ \\
\hline Miguel Pereira & 0,1951 & 0,16763 & $-0,57086$ & $-0,30372$ \\
\hline Paracambi & $-0,61946$ & $-0,39746$ & 0,13849 & 0,63872 \\
\hline Paty do Alferes & $-0,20444$ & $-0,32456$ & 0,71109 & 0,14478 \\
\hline Vassouras & 0,54013 & $-0,51344$ & 0,03379 & $-0,27437$ \\
\hline Bom Jesus do Itabapoana & 0,25491 & 0,45503 & $-0,04761$ & 0,06852 \\
\hline
\end{tabular}




\begin{tabular}{|c|c|c|c|c|}
\hline \multirow{2}{*}{ Municípios } & \multicolumn{4}{|c|}{ Escores } \\
\hline & $F 1$ & $F 2$ & F3 & F4 \\
\hline Itaperuna & 0,25396 & $-0,94326$ & $-0,42063$ & $-0,40492$ \\
\hline Laje do Muriaé & $-1,08086$ & $-0,40801$ & $-0,38665$ & $-0,01072$ \\
\hline Natividade & 1,04262 & 1,26236 & $-0,5944$ & 0,40227 \\
\hline Porciúncula & 0,24605 & $-0,64144$ & 0,24015 & $-0,14959$ \\
\hline Varre-Sai & 0,01478 & $-0,38356$ & $-0,18383$ & $-0,33856$ \\
\hline Aperibé & $-1,33782$ & $-0,12167$ & $-0,4911$ & $-0,3418$ \\
\hline Cambuci & $-0,32483$ & 1,90568 & $-0,49369$ & $-2,16277$ \\
\hline Itaocara & $-0,71126$ & $-0,89373$ & $-0,27467$ & 0,11313 \\
\hline Miracema & 0,27152 & 0,45845 & 0,06671 & 1,25598 \\
\hline Sto. Ant. de Pádua & $-0,26972$ & $-0,60032$ & $-0,156$ & 0,05528 \\
\hline Campos dos Goytacazes & 1,74312 & 0,15338 & 0,25543 & $-0,65351$ \\
\hline Cardoso Moreira & 0,75068 & 0,71798 & $-0,19101$ & 4,02059 \\
\hline São Fidélis & $-0,0879$ & 0,08607 & 0,14906 & 0,01917 \\
\hline São Francisco do Itabapoana & $-0,14505$ & 0,50345 & $-0,33895$ & $-0,51456$ \\
\hline São João da Barra & 0,53173 & 0,11695 & $-0,19259$ & $-0,07159$ \\
\hline Carapebus & 2,47985 & $-2,54948$ & $-1,66165$ & 0,54865 \\
\hline Conceição de Macabu & 3,31894 & 1,8995 & $-0,60978$ & 0,08903 \\
\hline Macaé & 0,26163 & 0,86148 & $-0,52178$ & 1,71433 \\
\hline Quissamã & 0,23903 & 0,13605 & 0,66734 & $-1,18367$ \\
\hline Barra do Piraí & $-0,80823$ & 0,06578 & $-0,43954$ & $-0,20643$ \\
\hline Rio das Flores & $-0,04119$ & 0,57474 & 0,29066 & $-0,16455$ \\
\hline Valença & $-0,31632$ & $-0,90194$ & 1,24113 & 0,942 \\
\hline Barra Mansa & $-0,55435$ & 0,3788 & 0,20731 & 0,27312 \\
\hline Itatiaia & 0,09686 & $-0,9479$ & $-0,54918$ & $-0,37441$ \\
\hline Piraí & $-0,34974$ & 0,27634 & $-0,35898$ & 0,45964 \\
\hline Quatis & $-0,51322$ & $-0,81268$ & 0,01291 & $-0,16679$ \\
\hline Resende & $-0,39384$ & $-0,14033$ & $-0,39678$ & 0,05984 \\
\hline Rio Claro & 1,89377 & 0,92554 & 0,15325 & 0,10113 \\
\hline Volta Redonda & $-0,59985$ & 0,46919 & 0,50624 & 1,35572 \\
\hline
\end{tabular}

Fonte: Dados da pesquisa, baseado no Censo Apícola do estado do Rio de Janeiro (2006).

A partir dos escores fatoriais de cada município, foram calculados os Índices Brutos de Desenvolvimento, mediante os quais os municípios foram classificados (Tabela 4). Foram observados índices brutos de desenvolvimento acima de 0,50 nos municípios de Conceição de Macabu, Areal, Duas Barras, Rio Claro, Cardoso Moreira, Cachoeiras de Macacu, Campos dos Goytacazes, Natividade e Nova Iguaçu. Foi verificado que o grau de escolaridade está relacionado ao desenvolvimento dos apicultores. O nível de analfabe- tismo nos apicultores destes municípios foi zero e, mais de $40 \%$ dos apicultores, em média, têm pelo menos o Ensino Médio completo. Outras características que contribuíram para elevar esse índice nos referidos municípios foram: assistência técnica (Nova Iguaçu com 100\% e Natividade com $48 \%$ ); o manejo da troca de rainha (Nova Iguaçu com $100 \%$ e Cardoso Moreira com $47,5 \%$ ) e a prática da apicultura migratória (Nova Iguaçu com 100\%, Cachoeiras de Macacu com 75\% e Conceição de Macabu com 33\%). 
Tabela 5. Classificação dos municípios pelos valores do Índice Bruto de Desenvolvimento

\begin{tabular}{|c|c|c|c|}
\hline Municípios & Índice & Municípios & Índice \\
\hline Conceição de Macabu & 1,810 & Petrópolis & 0,137 \\
\hline Areal & 1,596 & Volta Redonda & 0,110 \\
\hline Duas Barras & 1,316 & Quissamã & 0,110 \\
\hline Rio Claro & 1,103 & Vassouras & 0,103 \\
\hline Cardoso Moreira & 0,997 & Valença & 0,030 \\
\hline Cachoeiras de Macacu & 0,950 & Mangaratiba & 0,017 \\
\hline Campos dos Goytacazes & 0,786 & São Fidélis & 0,011 \\
\hline Natividade & 0,684 & Porciúncula & $-0,001$ \\
\hline Nova Iguaçu & 0,542 & Paty do Alferes & $-0,005$ \\
\hline Rio das Ostras & 0,468 & Miguel Pereira & $-0,028$ \\
\hline Macaé & 0,433 & Rio Bonito & $-0,068$ \\
\hline Seropédica & 0,432 & Tinguá & $-0,075$ \\
\hline Carmo & 0,408 & Sapucaia & $-0,085$ \\
\hline Miracema & 0,404 & Itaguaí & $-0,090$ \\
\hline Niterói & 0,344 & S. Francisco do Itabapoana & $-0,092$ \\
\hline Carapebu & 0,319 & Barra Mansa & $-0,092$ \\
\hline Cabo Frio & 0,315 & Três Rios & $-0,107$ \\
\hline Japeri & 0,304 & Piraí & $-0,107$ \\
\hline Paraíba do Sul & 0,290 & Cambuci & $-0,120$ \\
\hline Comendador Levy Gasparian & 0,280 & Maricá & $-0,120$ \\
\hline Casimiro de Abreu & 0,258 & Piraí & $-0,107$ \\
\hline São João da Barra & 0,219 & Cambuci & $-0,120$ \\
\hline Bom Jesus do Itabapoana & 0,214 & Maricá & $-0,120$ \\
\hline Rio de Janeiro & 0,190 & Varre-Sai & $-0,158$ \\
\hline Saquarema & 0,165 & Itaboraí & $-0,170$ \\
\hline Rio das Flores & 0,141 & Engo Paulo de Frontin & $-0,175$ \\
\hline Municípios & Soma & Municípios & Soma \\
\hline Mendes & $-0,215$ & Duque de Caxias & $-0,568$ \\
\hline Itaperuna & $-0,226$ & Iguaba Grande & $-0,591$ \\
\hline Paracambi & $-0,255$ & Nova Friburgo & $-0,607$ \\
\hline Trajano de Morais & $-0,270$ & Santa Maria Madalena & $-0,612$ \\
\hline Sto. Ant. de Pádua & $-0,276$ & Cordeiro & $-0,644$ \\
\hline Resende & $-0,279$ & Laje do Muriaé & $-0,656$ \\
\hline Itatiaia & $-0,319$ & São Gonçalo & $-0,680$ \\
\hline Silva Jardim & $-0,346$ & Magé & $-0,761$ \\
\hline Araruama & $-0,393$ & Aperibé & $-0,775$ \\
\hline Quatis & $-0,429$ & Sumidouro & $-0,841$ \\
\hline Barra do Piraí & $-0,466$ & Teresópolis & $-0,874$ \\
\hline São Pedro da Aldeia & $-0,485$ & Macuco & $-0,933$ \\
\hline Itaocara & $-0,555$ & Bom Jardim & $-0,938$ \\
\hline
\end{tabular}

Fonte: Dados da pesquisa, baseado no Censo Apícola do estado do Rio de Janeiro (2006). 
Por outro lado, os municípios que apresentaram índice bruto de desenvolvimento abaixo de $(-0,50)$ foram: Itaocara, Duque de Caxias, Iguaba Grande, Nova Friburgo, Santa Maria Madalena, Cordeiro, Laje do Muriaé, São Gonçalo, Magé, Aperibé, Sumidouro, Teresópolis, Macuco e Bom Jardim. Alguns destes municípios ainda têm apicultores analfabetos, a saber: Nova Friburgo com 11,1\% e São Gonçalo com 7,7\%. Há muitos apicultores de outras atividades, inclusive 17,6\% com cursos superiores em áreas não agrárias. Assim, acredita-se que muitos destes têm baixa eficiência na produção de mel.

Os resultados dos principais indicadores do nível tecnológico explicam o baixo desempenho da produção de mel e a incapacidade de diversificação dos produtos apícolas explorados na maioria dos municípios do estado do Rio de Janeiro. A assistência técnica bem coordenada pode ser considerada um dos principais indicadores para elevar o nível tecnológico na produção agrícola. A importância deste serviço é fundamental na transferência de tecnologia nesta atividade de pequenos produtores.

O manejo da troca de rainhas foi relativamente baixo na maioria dos municípios, excetuando-se São João da Barra, Nova Iguaçu, Majé, Teresópolis e Petrópolis, que se destacaram com mais de $60 \%$ no uso desta tecnologia. A prática de apicultura migratória também se apresentou baixa na maioria dos municípios, com exceção de São Gonçalo (100\%), Cachoeiras de Macacu (75\%) e Tinguá (60\%). Essa migração, apesar de favorecer a produtividade, aproveitando o potencial florístico de outras áreas, não é muito utilizada pelas restrições de transporte e de mão de obra requeridas (Tabela 1A do Apêndice).

Verificou-se que houve elevada perda de colmeia em vários municípios, exceto para Saquarema, Duque de Caxias, Japeri e Nova Iguaçu, que não registraram prejuízos neste parâmetro. Este tipo de baixa nas colmeias pode estar relacionado ao manejo oferecido, como a localização do apiário e ao déficit de alimentação das abelhas em épocas de menor floração.

O trabalho utilizado de forma eficiente pode contribuir positivamente na produção final.
Observou-se na Tabela 1A do Apêndice que, em quase todos os municípios, a mão de obra disponibilizada foi abaixo de 100 dias por ano para a atividade apícola, com exceção de Mendes (198), Quissamã (179), Valença (179) e Niterói (178). O manejo correto das colmeias é importante, principalmente nas épocas que não coincidem com a coleta dos produtos e que frequentemente são negligenciadas pela maioria dos produtores. Dependendo da região, as visitas frequentes podem contribuir para o sucesso da atividade, principalmente identificando e eliminando problemas como doenças ou ataque de predadores, necessidade de troca de rainha, deslocamento de colmeias, entre outros.

O nível de escolaridade também influencia a adoção de tecnologias e facilita a tomada de decisões quanto ao tipo de exploração de produtos e os meios para otimizar sua produção. Observou-se nível baixo de escolaridade dos apicultores em quase todos os municípios, predominando maior porcentagem com apenas o Ensino Fundamental completo, incompleto ou alfabetização de adultos e cerca de 3\% de apicultores analfabetos. Dessa forma, a média de escolaridade apresentada pelos apicultores do estado foi de 54,3\% até o Ensino Fundamental, 28,4\% com Ensino Médio e 17,3\% com Ensino Superior.

\section{Conclusões}

O trabalho buscou caracterizar o nível tecnológico dos produtores de mel dos municípios do estado do Rio de Janeiro. Por meio da Análise Fatorial, aplicada a um conjunto de variáveis relacionadas ao emprego de tecnologias, foi possível concluir que o nível tecnológico dos apicultores é relativamente baixo. Por meio dos escores fatoriais calculou-se o índice bruto de desenvolvimento, que possibilitou classificar os municípios de acordo com o grau de adoção de tecnologia. Com valores mais altos desse índice, destacaram-se os municípios de Conceição de Macabu, Areal, Duas Barras, Rio Claro, Cardoso Moreira, Cachoeiras de Macacu, Campos, Natividade e Nova Iguaçu. 
Constatou-se que o grau de escolaridade influenciou positivamente o desenvolvimento dos apicultores. O nível de analfabetismo foi nulo para os apicultores destes municípios e grande parte deles tem pelo menos o Ensino Médio. Outras características que contribuíram para elevar esse índice nos referidos municípios foram assistência técnica, manejo da troca de rainha e a prática da apicultura migratória. Assim, a modernização dos apicultores no sentido de melhorar o nível tecnológico, expandir a produtividade e diversificar sua produção passa necessariamente pelo nível de conhecimento do apicultor e pela situação socioeconômica.

Como limitação do trabalho, acredita-se que, além dos fatores tecnológicos e de manejo apícola considerados no modelo, bem como do grau de escolaridade, também considerado na análise, existem outros fatores externos que não foram considerados e que podem ser limitantes na determinação do grau de desenvolvimento e de adoção de tecnologia. Como desafio para outros estudos, seria importante adicionar análises de indicadores sociais, de políticas de incentivos, de formas de organização e de comercialização da produção, com a finalidade de compreender a importância destes elementos numa análise sistêmica do universo apícola. Outra investigação pertinente seria verificar a adequação da assistência técnica e da extensão rural levada aos apicultores fluminenses, visando capacitação tecnológica e gerencial. A apicultura, para ser competitiva, exige a adoção de boas práticas de manejo, de equipamento adequado, de conhecimento específico de tecnologias, de gestão gerencial, de comercialização e de organização para agregar valores aos produtos.

\section{Referências bibliográficas}

BLAKE, C. e SALEH, S. D. A Model of Entrepreneurial Venture Performance, Journal of small business entrepreneurship, v. 4, n. 4, jul./set. 1992.

CARVALHO, D. F., SANTANA, A. C., NOGUEIRA, A. K. M., MENDES, F. A. T. e CARVALHO, A. C. Análise do desempenho competitivo da indústria de móveis de madeira do estado do Pará. Amazônia: Ci. E Desenv., Belém - PA, v. 2, n. 4, jan./jun. 2007.

CENSO APÍCOLA DO ESTADO DO RIO DE JANEIRO: Análise Conjuntural. Serviço Nacional de Aprendizagem do Cooperativismo no Rio de Janeiro. Ed. SNAC-RJ, 2008.

CHINELATTO NETO, A., CASTRO, G. P. C. e LIMA, J. E. Uso de análise estatística multivariada para tipificação de produtores de leite de Minas Gerais. Organizações Rurais \& Agroindustriais, 2005, 7. Disponível em: $<$ http://www.redalyc.org/src/inicio/ArtPdfRed. jsp?iCve $=87817147010>$. Acesso em: 8 jan. 2013.

FREITAS, D. G. F., KHAN, A. S. e SILVA, L. M. R. Nível tecnológico e rentabilidade de produção de mel de abelha (Apis mellifera) no Ceará. Revista de Economia e Sociologia Rural, Brasília - DF, v. 42, n. 1, p. 171-188, jan./mar. 2004.

HARMAN, H. H. Modern Factor Analysis. 2. ed. University of Chicago Press, Chicago, 1967.

IBGE. Banco de Dados Agregados. Sistema IBGE de Recuperação Automática-SIDRA. Disponível em $<$ http://www.sidra.ibge.gov.br/bda/tabela/listabl. asp? $\mathrm{c}=74 \& \mathrm{z}=\mathrm{t} \& \mathrm{o}=22>$. Acesso em: 21 nov. 2011.

IBGE. Banco de Dados Agregados. Sistema IBGE de Recuperação Automática-SIDRA. Disponível em: $\quad<$ http://sidra.ibge.gov.br/bda/tabela/protabl. asp? $c=74 \& z=p \& o=26 \& i=P>$. Acesso em: 02 dez. 2011.

JOHNSON, R. A. e WICHERN, D. W. Applied multivariate statistical analysis. 3. ed. (International Edition). Prentice Hall, 1992.

KHAN, A. S., MATOS, V. D. e LIMA, P. V. P. S. Desempenho da apicultura no estado do Ceará: Competitividade, nível tecnológico e fatores condicionantes. Revista de Economia e Sociologia Rural, Piracicaba, v. 47, n. 3, p. 651-675, 2009.

KIM, Jae-On e MUELLER, C. W. Introduction to factor analysis What it is and how to do it. Newbury Park, Califórnia: Sage Publications, 1978.

MELO, C. O. e PARRÉ, J. L. Índice de desenvolvimento rural dos municípios paranaenses: determinantes e hierarquização. Revista de Economia e Sociologia Rural, Brasília, v. 45, n. 2, p. 329-365, abr.jun. 2007.

MONTEIRO, V. P. e PINHEIRO, J. C. V. Critério para implantação de tecnologias de suprimentos de água potável em municípios cearenses afetados pelo alto teor de sal. Revista de Economia e Sociologia Rural, Brasília - DF, v. 42, n. 2, p. 365-387, abr.jun. 2004.

ROGERS, E. M. Diffusion of innovations. New York: The Free Press, 2003. 
SCHILDERINCK, J. H. F. Factor Analysis Applied to Developed and Developing Countries. Rotterdan University Press, Groningen. Wolters-Noordhoff, 1970.

SOUZA, D. C. A história da apicultura no Brasil. Disponível em: <http://www.sebrae.com.br/setor/ apicultura/sobre-apicultura/apicultura-no-brasil/ historia/ integra_bia/ident_unico/688> . Acesso em: 22 out. 2011.
SOUZA, P. M., PONCIANO, N. J., MATA, H. T. C., BRITO, M. N. e GOLINSKI, J. Padrão de desenvolvimento tecnológico dos municípios das Regiões Norte e Noroeste do Rio de Janeiro. Revista de Economia e Sociologia Rural. Brasília - DF, v. 47, n. 4, out./ dez. 2009.

TORNATZY, L. G. The Process of Technological Innovation, Lexington Books, Lexington, MA, 1990. 


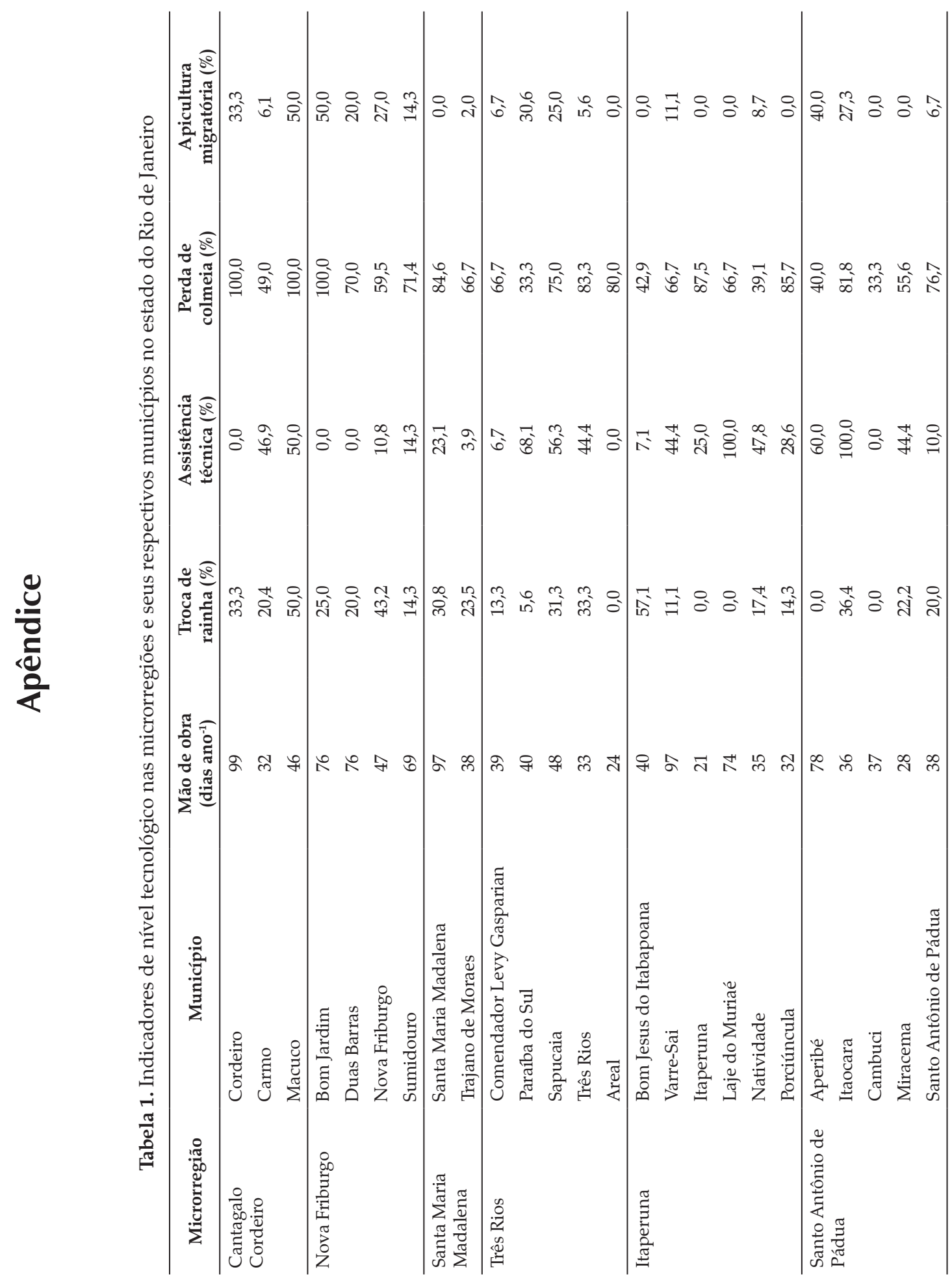




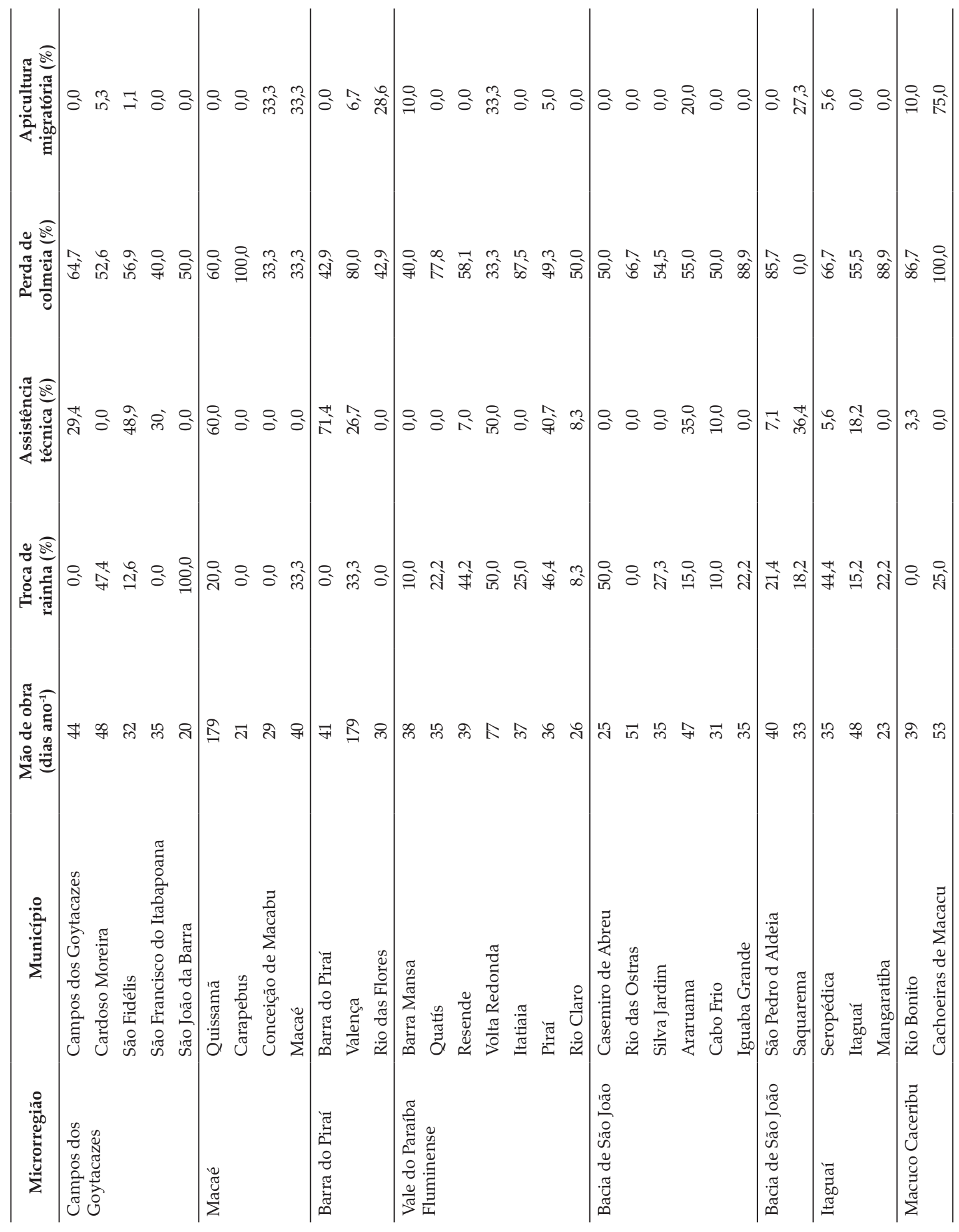




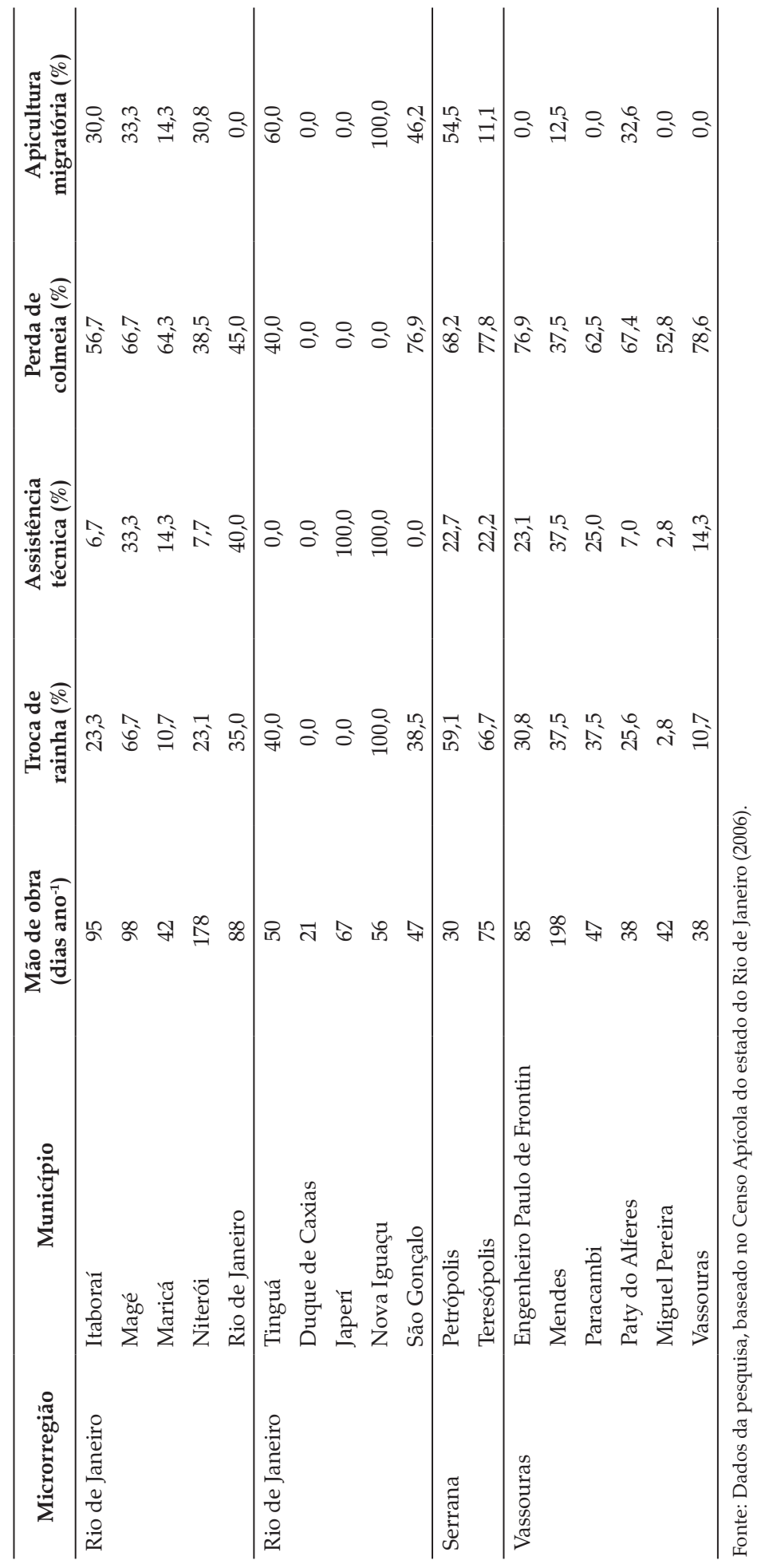

